\title{
What's the Connection between Diabetes and Depression?
}

\author{
Elif Ünsal Avdal ${ }^{1}$, Berna Nilgün Özgürsoy Uran ${ }^{1 *}$, Burcu Arkan ${ }^{1,2}$ and Funda Sofulu ${ }^{1}$ \\ ${ }^{1}$ Faculty of Health Science, Izmir KatipCelebi University, Izmir, Turkey \\ ${ }^{2}$ Faculty of Health Science, Uludag University, Bursa , Turkey
}

Submission: February 2, 2017; Published: May 24, 2017

*Corresponding author: Berna Nilgün Özgürsoy Uran, Faculty of Health Science, Izmir KatipCelebi University, Izmir, Turkey, Email: bernanilgun@gmail.com

\begin{abstract}
Depression is a serious medical condition that affects thoughts, feelings, and the ability to function in everyday life.

An internationalreport has shownthathavingdiabetesanddepression has thegreatestnegativeeffect on quality of life comparedtodiabetesordepressionalone, orotherchronicconditions. Because of tahatreasonIt is very important for health professionals to control these diseases to understand the relation of diabetes to depression. The purpose of this review is to draw attention to the relationship between depression and diabetes and to provide literature support to health professionals.
\end{abstract}

Keywords: Diabetes; Depression; Treatment

\section{Introduction}

The increasing burden of diabetes among adults (aged 20-70 years) is a major public health concern globally [1]. The number of people living with diabetes is estimated to rise from 135 million in 1995 to 300 million by 2025. Diabetes is a disorder that impairs the way the body uses digested food for growth and energy. Most of the food we eat is broken down into glucose, a form of sugar that provides the main source of fuel for the body. After digestion, glucose passes into the bloodstream. Insulin, a hormone produced by the pancreas, helps glucose get into cells and converts glucose to energy. Without insulin, glucose builds up in the blood, and the body loses its main source of fuel [1].

Type 1 diabetes is when the immune system destroys the insulin-producing beta cells of the pancreas. This form of diabetes usually strikes children and young adults, who require daily or more frequent insulin injections or using an insulin pump for the rest of their lives. Insulin treatment, however, is not a cure, nor can it reliably prevent the long-term complications of the disease. Although scientists do not know what causes the immune system to attack the cells, they believe that both genetic factors and environmental factors are involved [1].

Type 2 diabetes, which accounts for about 90 percent of diabetes cases in the United States, is most common in adults over age 40. Affecting about 6 percent of the U.S. population, this form of diabetes is strongly linked with obesity (more than 80 percent of people with type 2 diabetes are overweight), inactivity, and a family history of diabetes. It is more common in African Americans, Hispanic Americans, American Indians, and Asian and Pacific Islander Americans. With the aging of Americans and the alarming increase in obesity in all ages and ethnic groups, the incidence of type 2 diabetes has also been rising nationwide [1].Theprevalence of majordepression in the UK population at anyone time is about $5 \%$, although as many as oneperson in threemayexperience an episodeof depression in theirlifetime [2].

There search ersused cognitive therapy to reverse the depression.In those people where depression improved, there was an average $\mathrm{HbA1c}$ of $8.3 \%$ while those who showed little improvement had an average of $11.3 \%$. While these results show an association between highblood sugars and depression, it remains unclear whether highblood sugars cause the depression or depression causes highblood sugars [2].

Another relatively common and costly central nervous system syndrome, depression, occurs in many patients with diabetes [1]. Depression is a serious medical condition that affects thoughts, feelings, and the ability to function in everyday life. Depression results from abnormal functioning of the brain. The causes of depression are currently a matter of intense 
research. An interaction between genetic predisposition and life history appear to determine a person's level of risk. Episodes of depression may then be triggered by stress, difficult life events, side effects of medications, or other environmental factors. Whatever its origins, depression can limit the energy needed to keep focused on treatment for other disorders, such as diabetes [1].।

A more recent systematic review and meta-analysis of multiple community-based, large-scale, prospective studies confirmed the significantly increased rates of depression in people with type 2 diabetes relative to control subjects (17.6\%vs9.8\%, OR=1.6 [95\% confidence interval (CI) of 1.22.0]) [3]. Despite the differences between type 1 and type 2 diabetes in age of onset and clinical course, the prevalence rates for type 1 vs type 2 diabetes were statistically similar in the studies that determined depression by diagnostic interview (13.6\% vs10.9\%, $>0.1$ ) or with self-reportsymptoms scales (29.1\%vs32.9\%, p>0.1) [4].

Cardiovascular disease is a macro vascular complication of diabetes. An analysis of a large, naturalistic population-based sample of patients with type 2 diabetes in a Large primary care system concluded that patients with major depression and diabetes were 1.5-2-fold more likely to have 3 or more cardiovascular risk factors than patients with diabetes without depression [5].

\section{How Does Depression Affect People with Diabetes?}

An international report has shown that having diabetes and depression has the greatest negative effect on quality of life compared to diabetes or depression alone, or other chronic conditions (Table 1).

Table 1:The negative impact of depression upon patients with diabetes Rubin et al. [6].

\begin{tabular}{|c|}
\hline Worsening glycemic control \\
\hline $\begin{array}{c}\text { Increase in severity or number of diabetic complications such as } \\
\text { retinopathy, sexual dysfunction, and nephropathy }\end{array}$ \\
\hline Increased likelihood of cardio vascular risk factors \\
\hline Higher rates of functional disability \\
\hline Higherall-cause mortality \\
\hline
\end{tabular}

\section{Though the relationship between diabetes and depression isn't fully understood}

a) The rigors of managing diabetes can be stressful and lead to symptoms of depression.

b) Diabetes can cause complications and health problems that may worsen symptoms of depression.

c) Depression can lead to poor lifestyle decisions, such as unhealthy eating, less exercise, smoking and weight gain - all of which are risk factors for diabetes. d) Depression affects your ability to perform tasks, communicate and think clearly.

e) This can interfere with your ability to successfully manage diabetes.

Recent estimates suggest that upto three quarters of cases of depression in people with diabetes may go undiagnosed. During the subsequent decade of 2000-2010,increased Attention focused upon adverse metabolic and thrombo vascularc onsequences of depression through certain biologic path ways.

Relevant in this regard are the hypothalamic-pituitaryadrenal axis hyperactivity and mental stress-induced sympatho medullary activation in many patients with major depression, which can contribute to decreased glucose transport and insulinresistance, respectively.A mechanistic path way between depression, diabetes,and coronary artery disease has been proposed by Steven Roose [7].

Depression causes patients to become inactive, which, combined within creased cortisol levels, causes adiposity, obesity, and insulin resistance. Adipose tissue and damaged vessels then release pro-inflammatory cytokines, which induce the depressive symptoms of the "sicknessbehavior"syndrome, which impair a patient's motivation to initiate healthy self-care behaviors and perturb cognition. Consequently,ever-worsening cycles evolve eventually resulting in a heartattack or stroke associated with type 2 diabete [7].

\section{Treatment Depression and Diabetes}

There are multiple conceptual approaches to the diagnosis of major depression in medically ill patients, including the exclusive and inclusive approaches [8]. The exclusive approach maximizes specificity bye liminating so maticsymptoms such as anorexia and fatigue, which frequently are elements of medical disorders [8]. The inclusive approach includes all depressive symptoms to diagnose major depressive disorder in the medically ill and maximizes sensitivity, best protecting the patient from a "falsenegative result" and the risk of undiagnosed depression [8].

Non-depressed diabetic patients may report neurobehaviora symptoms such as diminished appetite, weightloss, hypersomnia, loss of libido and psycho motor retardation [9]. Therefore, the use of exclusive criteria may allow clinicians to more easily identify major depression in diabetic patients [9].

Treatment for depression in people with diabetes has been shown to be effective and has the additional benefits of improving blood sugar control. The evidence suggests that cognitive behaviour therapy and anti-depressant medicines are as effective in people with diabetes as in those with out diabetes. As thetreatment of depression can improve blood sugar control, it is also likely to reduce the risk of complications but importantly, it can also improve quality of life and diabetes self management programs, psychotherapy, medications and lifestyle changes, 
collaborative care is very important to treatment depression and diabetes (Table 2).

Table 2: Treatment Depression and Diabetes $[1,10]$.

\begin{tabular}{|c|}
\hline $\begin{array}{l}\text { lat } \\
\text { ove } \\
\text { ght } \\
\text { help }\end{array}$ \\
\hline $\begin{array}{l}\text { Psychotherapy: Similarly, participants in psychotherapy, particularly } \\
\text { cognitive behavioral therapy, have reported improvements in } \\
\text { depression, which has resulted in better diabetes management. }\end{array}$ \\
\hline $\begin{array}{l}\text { Medications and lifestyle changes: Medications for both diabetes } \\
\text { and depression and lifestyle changes, including different types of } \\
\text { therapy coupled with regular exercise, can improve both conditions. }\end{array}$ \\
\hline $\begin{array}{c}\text { Collaborative care: New research shows that treatment supervised } \\
\text { by a nurse case manager that steps up therapy when needed helps } \\
\text { improve both depression and diabetes. This type of care may not be } \\
\text { available in most health care systems. }\end{array}$ \\
\hline
\end{tabular}

Physicians always need to be cognizant of drug-drug interactions when prescribing medications, particularly in patientswith psychiatric and medical co-morbidities. Due to the inhibition of cytochrome P450 (CYP) 3A4 isoenzyme,certain of these rotoninre uptake inhibitors (SSRIs) such as fluoxetine and fluvoxamine mayalter the metabolism of certain oral hypoglycemics (such as the thiazolidinedione pioglitazone, the meglitinies, repaglinide and nateglinide) and confer a risk of hypoglycemia [9].

In addition, inhibition of the CYP 2C9 isoenzyme by fluoxetine, fluvoxamine, or sertraline may also potentially alter CYP 2C9 metabolism of sulfonylureas tolbutamide and glimepiride [9]. Diabetes nurses can say diabetes self management progrmas is very important to treating depression and diabetes [1].

\section{Conclusion}

There is clearly an increased prevalence of depression among diabetic patients [1]. Depressive symptoms serve as a risk factor for the development of diabetes and have been shown to contribute to hyperglycemia, diabetic complications, functional disability and all cause mortality among diabetic patients. Despite the fact that depression complicates medical conditions such as diabetes, there is evidence that diabetes nurse and primary care physicians treat depression less aggressively in patients with medical comorbidities [11].

\section{References}

1. Santos SA, Alberto SC, Cruz E, Pires E, Figueira T, et al. (2007) Hepatic abscess induced by foreign body: Case report and literature review. World Journal of Gastroenterology 13(9): 1466-1470.

2. Chen HK, Kuo JR, Uen YH, Sun DP (2011) Liver abscess secondary to fish bone migration from the duodenum 81(3): 206.

3. Kendra K, Walter P, Yi-Horng L (2012) Transluminal migration of ingested foreign body without peritonitis. Journal of Pediatric Surgery 47(4): 788-791.

4. Dominguez S, Wildhaber BE, Spadola L, Mehrak AD, Chardot C (2009) Laparoscopic extraction of intra hepatic foreign body after transduodenal migration in a child. Journal of Pediatric Surgery 44(11): E17-E20.

5. Aarabi S, Stephenson J, Christie DL, Javid PJ (2012) Noningested intraperitoneal foreign body causing chronic abdominal pain: a role for laparoscopy in diagnosis. Journal of Pediatric Surgery 47 (20: E15-E17.

6. Rodríguez-Hermosa JI, Codina-Cazador A, Sirvent JM, Martín A, Gironès J, et al. (2008) Surgically treated perforations of gastrointestinal tract caused by ingested foreign bodies. Colorectal Disease 10(7): 701-707.

7. Goh BK, Chow PK, Quah HM, Ong HS, Eu KW, et al. (2006) Perforation of gastrointestinal tract secondary to ingestion of foreign bodies. World J Surg (30): 372-377.

8. Rodrigues D, Perez NE, Hammer PM, John DW (2006) Laparoscopic removal of a retained intra-abdominal ribbon malleable retractor after 14 years. J Laparoendosc Adv Surg Tech 16: 369-371.

9. Le Mandat-Schultz A, Bonnard A, Belarbi N, Aigrain Y, Lagausie PD (2003) Intrahepatic foreign body laparoscopic extraction. Surg Endosc 17(11): 1849.

10. Omejc M (2002) Laparoscopic removal of an ingested pin migrating into the liver. Surg Endosc 16(3): 537.

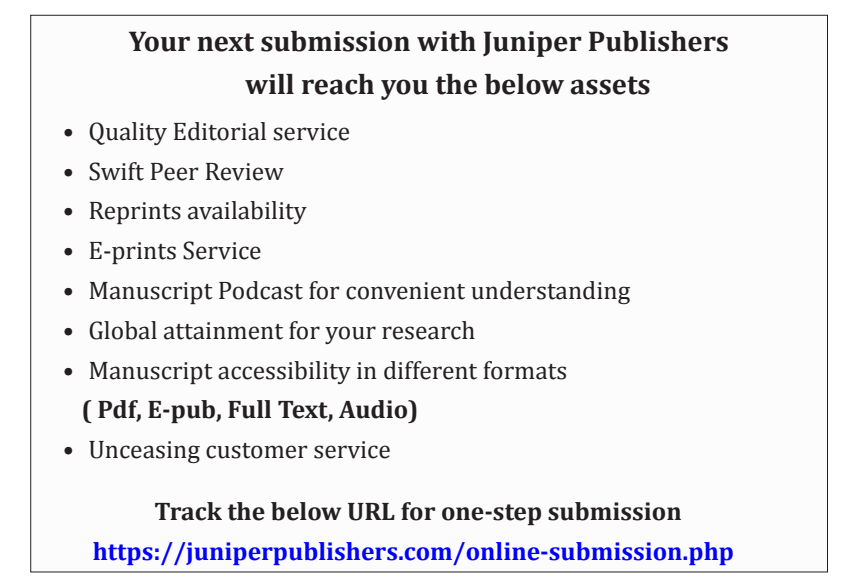

